\title{
Evaluación de la producción potencial de frutos de Gevuina avellana, durante una temporada (1999-2000) en el secano costero de la VII Región*
}

Potential fruit production evaluation of Gevuina avellana, during one season (1999-2000) in the coastal dryland of the VII Region

\section{URSULA DOLL ${ }^{1}$, JOSE SAN MARTIN ${ }^{2}$, CARLOS RAVANAL ${ }^{3}$,} SANDRA CIFUENTES, MONICA MUÑOZ

\footnotetext{
${ }^{1}$ Universidad de Talca, Departamento de Gestión Forestal Ambiental, Casilla 721-747, Talca, Chile. E-mail: udoll@utalca.cl

${ }^{2}$ Universidad de Talca, Instituto de Biología Vegetal y Biotecnología, Casilla 721-747, Talca, Chile, E-mail: jsanmart@utalca.cl

${ }^{3}$ Corporación Nacional Forestal, Región Metropolitana, Eliodoro Yáñez N¹810, Providencia, Santiago, Chile. E-mail: cravanal@conaf.cl
}

\begin{abstract}
SUMMARY
Gevuina avellana Mol., belongs to a monospecific genera of the Proteaceae family, which is endemic to Chile and Argentina. This evergreen Chilean tree is a component of different native habitats from the Andes and the Coastal mountains, between the VII and the X Region. This species has a wide range of uses, the most outstanding of which is its production of edible nuts. Traditionally the Chilean hazelnuts were collected from the native forest and marketed locally. Their excellent properties converted them into a fruit-species with great economic potential. In order to respond to quantity and quality demands of the chocolate industry, the potential production of Chilean hazelnuts was studied in the coastal dryland in the VII Region, using traps to collect samples. Based on their dimensions, insertion into the surrounding vegetation, and anthropic management, five tree types were defined. The greatest productivity was found on isolated trees surrounded by periodically fertilized and irrigated crops, with a production of nearly $14 \mathrm{~kg} / \mathrm{tree}$. This indicated the possibility of incorporating avellana into silvoagricultural management. Nut drop is concentrated in a period of 5 to 6 weeks, with the possibility of scheduling the harvest to minimize production and quality losses. It was observed that marked flower clusters need 8 months between the formation and ripening of nuts, with 1.6 to $4.7 \%$ of fruit set depending on the tree type.
\end{abstract}

Key words: Chilean hazelnut, Gevuina avellana Mol., non-wood products.

\section{RESUMEN}

Gevuina avellana Mol., Proteaceae, es una especie de un género monoespecífico, endémica de Chile y Argentina. Con hábito arbóreo siempreverde, forma parte de los bosques nativos de ambas cordilleras de Chile, entre las VII y X Regiones. Dentro de las amplias posibilidades de usos de la especie, se destaca su producción de frutos comestibles o "avellanas", las cuales se colectan y comercializan a nivel local. Sus excelentes propiedades comestibles la posicionan como un gran potencial frutícola y económico. Para responder a exigencias de cantidad y calidad para la industria de chocolatería, se estudió la producción potencial de frutos de avellanas

* Trabajo de Investigación financiado por FIA, Proyecto N V97-0-F001. 
en una localidad del secano costero de la VII Región, utilizando un sistema de muestreo por trampas. En base a su tamaño, inserción en la vegetación circundante y manejo antrópico, se definieron cinco tipos de árboles de avellanos. La mayor productividad se registró en árboles aislados y rodeados de cultivos suplementados con riego y fertilizaciones periódicas, con un promedio aproximado de $14 \mathrm{~kg} / a$ rbol. Este hecho sugiere la posibilidad de incorporarlo a sistemas de manejos silvoagrícolas. Se observó que la caída de frutos se concentra en un período de 5 a 6 semanas, permitiendo una programación de la cosecha con el fin de minimizar pérdidas y mermas de calidad. Se siguió el desarrollo de frutos en racimos marcados, observando que entre la aparición de los primeros frutos hasta su maduración transcurren 8 meses, con un porcentaje de cuaje que varía entre 1,6 y 4,7\% en los distintos tipos de árboles de avellanos.

Palabras clave: avellanas, Gevuina avellana, no maderables.

\section{INTRODUCCION}

El avellano (Gevuina avellana Mol.) es un árbol siempreverde endémico de Chile y Argentina, que pertenece a un género monoespecífico de la familia Proteaceae. En Chile, entre las VII y XI Regiones $\left(35^{\circ}\right.$ a $44^{\circ}$ latitud sur (1)), esta especie integra los bosques nativos de las Cordilleras de los Andes y de la Costa (2, 3). Fisionómicamente se destaca su follaje verde brillante, así como sus brotes nuevos con un tomento ferrugíneo (4). Las flores pequeñas, blanco cremosas, se disponen en largos racimos axilares de polinización entomófila (5). El fruto es una nuez indehiscente, con pericarpio leñoso y semilla comestible que en su desarrollo presenta cambios de coloración de verde a rojo y finalmente pardo oscuro en la madurez (4).

Gevuina avellana presenta múltiples usos como maderera, melífera y ornamental, destacándose la producción de frutos aptos para la industria cosmética, farmacológica y de alimentos $(5,6)$. La similitud de sus nueces con las de Macadamia spp. (Proteaceae originaria de Australia, difundida como frutal de nuez para clima tropical y subtropical) ha despertado el interés del mercado internacional como una alternativa para climas templados (7).

Tradicionalmente, las avellanas o nueces se recolectan del bosque nativo para comercializarlas a nivel local. Esta actividad representa una fuente de ingreso alternativa, especialmente para familias rurales del secano costero de la VII Región. La posibilidad de utilizar la avellana en la industria de chocolatería contribuiría en un mejoramiento sustancial del ingreso familiar. Sin embargo, las fluctuaciones en la producción y el desconocimiento del proceso productivo en sí de esta especie la ubican actualmente como actividad riesgosa y poco rentable. Los antecedentes disponibles sobre la producción de frutos se han obtenido para el área sur de su distribución $(1,8,9)$, existiendo una evaluación anual de la producción de avellanas en una plantación de 20 años de edad en el Fundo Las Palmas de la Universidad Austral, Valdivia $(5,10)$. Por otro lado, se dispone de información informal sobre mayores potenciales de producción para la zona norte de su distribución. En este contexto, el objetivo del estudio se centró en obtener una aproximación del potencial productivo de avellanas en el secano costero de la VII Región de Chile, caracterizando los árboles en función de su tamaño y uso actual. A tal fin se cuantificó la producción individual de los árboles y las dimensiones de sus frutos, así como el proceso productivo, en cuanto a la transformación de flores en frutos y al tiempo de maduración y caída de los mismos.

\section{MATERIAL Y METODOS}

Area de estudio. El estudio se desarrolló en Salto

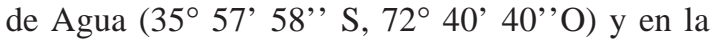
Reserva Nacional Los Queules ( $35^{\circ} 58^{\prime} \mathrm{S}, 72^{\circ}$ 41' O), ubicadas en la Cordillera de la Costa, de la Comuna de Pelluhue, provincia de Cauquenes (VII Región).

Selección de los árboles. En el bosque caducifolio maulino (11) se seleccionaron avellanos con distinto grado de intervención y manejo. Teniendo en cuenta esta heterogeneidad y en base a su tamaño, inserción en la vegetación circundante y manejo antrópico, se definieron los siguientes cinco tipos de árboles:

- tipo 1: productivos grandes: árboles de gran tamaño, aislados y rodeados de cultivos agrí- 
colas o pasturas fertilizadas y regadas periódicamente

- tipo 2: productivos medianos: árboles de tamaño intermedio a pequeño, aislados con vegetación nativa circundante degradada y sometida a pastoreo extensivo

- tipo 3: grandes de bosque: árboles grandes, insertos en un bosque degradado, formando parches de varios individuos cuyas copas se entremezclan. Periódicamente el propietario limpia el sotobosque

- tipo 4: reserva: árboles maduros, aislados e insertos en el bosque de la Reserva Nacional Los Queules

- tipo 5: renovales: árboles pequeños (renovales), rodeados de vegetación nativa intervenida y sin manejo actual.

A excepción de los árboles de tipo 4 de la Reserva Nacional de Los Queules, todos los demás tipos se caracterizan por presentar varios fustes principales. Este modelo de crecimiento, al parecer, indica un origen de rebrotes por tocón. La intervención antrópica y corta selectiva observada en algunos remanentes de bosque nativo del sector, apoyan este argumento.

Para el estudio y dependiendo de la accesibilidad, se eligieron entre 4 y 8 árboles por tipo, los que fueron controlados semanalmente para cuantificar su producción.

Caracterización de los árboles. Los árboles seleccionados se caracterizaron en base al DAP de todos los fustes principales de cada individuo obteniéndose un valor promedio. Luego, centrados en el fuste y en dirección norte-sur y esteoeste, se midió la cobertura o proyección de la copa, ubicando con coordenadas polares los puntos extremos de la copa. Esta información se traspasó a un plano a escala para calcular la superficie de cobertura proyectada por cada árbol.
Estimación de la producción de frutos. Debido al tamaño, forma y peso de los frutos de avellana, su caída es perpendicular al suelo y bajo la copa del árbol. La diseminación posterior es por gravedad a favor de la pendiente (12). Por ello se estimó la producción de frutos en base a un muestreo con trampas $(13,14,15)$, utilizando cajas de cartón de 0,6 m x 0,4 m de base y 0,5 $\mathrm{m}$ de altura. Estas trampas se distribuyeron en forma anidada alrededor del fuste del árbol en las cuatro direcciones cardinales y distanciadas a $1 \mathrm{~m}$ entre ellas. En los árboles de mayor envergadura se agregaron cuatro líneas equidistantes entre las anteriores. En todos los casos se aseguró una superficie equivalente al menos a un $10 \%$ de la proyección de la copa.

En algunos árboles aislados se instaló una malla Raschel alrededor del tronco y a $50 \mathrm{~cm}$ desde el suelo, para colectar la totalidad de los frutos. En el caso de los árboles del tipo 5 (renovales), los frutos se contaron en pie y previo al inicio de su caída.

Durante febrero a abril 1999 se recolectaron semanalmente los frutos de las trampas y las mallas. Cada muestra se pesó independientemente para obtener su peso fresco y se transportó a laboratorio en envase de papel.

Mediciones en laboratorio. Cada muestra fue secada en estufa de aire forzado a $45^{\circ} \mathrm{C}$ hasta peso constante (aproximadamente 1 semana) para determinar su peso seco. Paralelamente se midió el calibre (diámetros mayor y menor) en una submuestra seca de 10 avellanas por muestra.

Procesamiento de la información. Para estimar la producción total de frutos por árbol, se corrigieron los valores del peso seco de los frutos recogidos de las trampas mediante un factor de corrección [1], el cual relaciona la superficie de muestreo (superficie cubierta por trampas) con la proyección de copa para cada árbol:

$$
\text { factor de corrección } \text { (árbol } n)=\frac{\text { proyección de la copa }_{(a ́ r b o l ~ n)}}{\text { superficie cubierta por trampas } \text { tárbol } n)}
$$

Para estimar el volumen del fruto, se calculó el volumen de una esfera cuyo diámetro resulta del promedio entre los diámetros mayor y menor de la avellana.
Diseño experimental y análisis estadístico. Se utilizó un modelo unifactorial no balanceado. Los resultados para cada variable se sometieron a un análisis de varianza, contrastándose posteriormente 
las medias mediante la Prueba de Rangos Múltiples de Tukey, con nivel de probabilidad $p \leq 0,05$. Para homogeneizar la varianza se utilizó la transformación logarítmica. Para su evaluación, los datos expresados en porcentaje fueron modificados mediante la transformación angular.

Seguimiento de la transformación de flores en frutos. Se eligieron cinco ejemplares de los árboles del tipo 2 (productivos medianos), del tipo 3 (grandes de bosque) y del tipo 5 (renovales) respectivamente. En cada uno de ellos y a la altura de 1,60 m sobre el nivel del suelo se marcaron cuatro ramas. Cada una de las ramas seleccionadas y cada racimo floral en las mismas se enumeraron e identificaron con etiquetas de aluminio (16). Entre junio 1999 y febrero 2000 se registró mensualmente el número de flores y/o frutos en cada uno de los racimos marcados.

\section{RESULTADOS Y DISCUSION}

Caracterización de los árboles. Según el tamaño de la copa pueden diferenciarse dos grupos de árboles (cuadro 1): los de mayores dimensiones, que corresponden a los árboles grandes de bosque (tipo 3) con una proyección de copa promedio de $55,47 \mathrm{~m}^{2}$ y los más pequeños del grupo de los productivos medianos y renovales (tipos 2 y 5) con una proyección de copa promedio de 10,06 y $4,60 \mathrm{~m}^{2}$ respectivamente. Los árboles productivos grandes y los de la reserva (tipos 1 y 4) no difieren significativamente de los anteriores con proyecciones de copa promedio de 45,35 y $12,75 \mathrm{~m}^{2}$ respectivamente.

Respecto del DAP, es posible caracterizar tres grupos de árboles (cuadro 1): el primer grupo corresponde a los árboles productivos grandes, de la reserva y grandes de bosque (tipos 1, 4 y 3) con un DAP promedio de 22,44, 19,58 y $15,59 \mathrm{~cm}$ respectivamente. Un segundo grupo corresponde a los árboles productivos medianos (tipo 2) con un DAP promedio de $8,8 \mathrm{~cm}$ y finalmente los renovales (tipo 5) con un promedio de $2,5 \mathrm{~cm}$ de DAP.

Al superponer las variables copa y DAP, es posible diferenciar por un lado los árboles de grandes dimensiones, productivos grandes y grandes de bosque (tipo 1 y tipo 3), y por otro lado, los árboles de pequeñas dimensiones, productivos medianos y de renovales (tipo 2 y tipo 5). Los árboles de tipo 1 versus 3 y los árboles de tipo 2 versus 5 se diferencian adicionalmente por el grado de intervención actual de la vegetación acompañante, correspondiendo los de tipo 1 y 2 a la mayor intensidad de alteración, con cultivos y pastoreo respectivamente. Los árboles de la reserva (tipo 4) se caracterizan por su copa relativamente pequeña y un gran fuste.

\section{CUADRO 1}

Dimensiones promedio de los distintos tipos de árboles estudiados.

Mean dimensions of the different tree types studied.

\begin{tabular}{|ccccc|}
\hline $\begin{array}{c}\text { Tipo de árbol } \\
(*)\end{array}$ & $\begin{array}{c}\text { Número } \\
\text { de árboles } \\
\text { medidos }\end{array}$ & $\begin{array}{c}\text { Proyección } \\
\text { de la copa } \\
\left(\mathrm{m}^{2}\right)\end{array}$ & DAP $(\mathrm{cm})$ & (rango) \\
\hline 1 & 7 & $45,35 \mathrm{ab}$ & $22,44 \mathrm{a}$ & $(30,5-16,5)$ \\
2 & 5 & $10,06 \mathrm{c}$ & $8,80 \mathrm{~b}$ & $(13,8-6,7)$ \\
3 & 8 & $55,47 \mathrm{a}$ & $15,59 \mathrm{a}$ & $(19,2-11,0)$ \\
4 & 4 & $12,75 \mathrm{bc}$ & $19,58 \mathrm{a}$ & $(26,0-14,0)$ \\
5 & 5 & $4,60 \mathrm{c}$ & $2,50 \mathrm{c}$ & $(3,0-1,5)$ \\
\hline
\end{tabular}

(*) 1: productivos grandes; 2: productivos medianos; 3: grandes de bosque; 4: reserva; 5: renovales. Los valores seguidos de distintas letras presentan diferencia estadísticamente significativa según Prueba de Rangos Múltiples de Tukey, con $\mathrm{P} \leq 0,05$. 


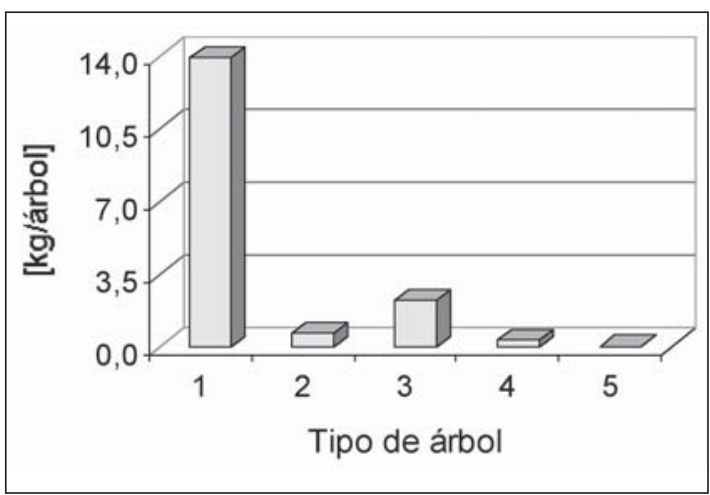

Figura 1. Producción de frutos de los 5 tipos de árboles estudiados $(*)$.

Fruit production of 5 tree types studied $(* *)$.

(*) 1: productivos grandes; 2 : productivos medianos; 3: grandes de bosque; 4: reserva; 5: renovales.

(**) 1: large sized productive; 2: medium sized productive; 3: large forest trees; 4: reserve trees; 5 : young trees.

Producción de frutos. Los árboles productivos grandes (tipo 1) registraron las mayores producciones de frutos durante la temporada 1999, con un promedio de 13,94 kg/árbol (figura 1). Le siguen los árboles grandes de bosque, productivos medianos y de reserva (tipos $3,2,4$ ) con 2,22, 0,68 y 0,31 kg/árbol respectivamente, mientras que los renovales (tipo 5) alcanzaron un promedio de sólo $34 \mathrm{~g}$ por árbol. La producción de los árboles productivos grandes (tipo 1) supera los valores promedio de 4,96 y 7,84 kg por árbol, reportados para avellanos de similares dimensiones de DAP (16 a $30 \mathrm{~cm}$ ) para dos localidades de la XI Región $(1,8)$. Por otro lado, una plantación iniciada 1983 en Valdivia (X Región), con material no seleccionado y a una densidad de 625 plantas por hectárea (5), alcanzó a los 16 años de edad una producción prome- dio de alrededor $5 \mathrm{~kg}$ por árbol $(10,17)$, detectándose una variabilidad entre plantas y años. Similar situación se observó entre árboles del área de Cauquenes (cuadro 2) a lo que, según información de lugareños, se suman fluctuaciones interanuales. Sin embargo, los árboles agrupados dentro de la categoría de productivos grandes se caracterizan por producciones sobresalientes año tras año. Cabe destacar que 5 de $\operatorname{los} 7$ árboles productivos grandes medidos superaron los $10 \mathrm{~kg}$ de producción por árbol.

Una plantación iniciada en 1987, en Valdivia (X Región), con 11 clones seleccionados, a los 10 años de edad logró una producción promedio de $20,04 \mathrm{~kg}$ por planta (6) y de $21,99 \mathrm{~kg}$ por árbol para 6 clones superiores $(17,18)$. Este resultado supera ampliamente la producción in situ de nuestro estudio. Sin embargo, teniendo en cuenta los rangos reportados de $0,9-28 \mathrm{~kg}$ por árbol en el caso de la plantación con material no seleccionado y 8,07 - 33,72 para los 6 clones superiores (17), puede inferirse que, en la zona central, es posible lograr resultados similares con un plan de selección, ya que en el caso de los árboles de tipo 1 (productivos grandes) se midió un rango de 1,24 a 44,35 kg por avellano (cuadro 2).

Hay que destacar que los altos valores individuales alcanzados corresponden a árboles de grandes dimensiones (cuadro 1). Por lo tanto, para descartar la influencia del tamaño del árbol en la producción individual se analizaron los datos mediante un análisis de covarianza, comportándose la variable "proyección de la copa" como covariante estadísticamente significativa $(\mathrm{p} \leq 0,05)$. A raíz de ello se definió la "productividad de la copa" [2] con la finalidad de expresar la producción de cada árbol en función de la proyección de su copa:

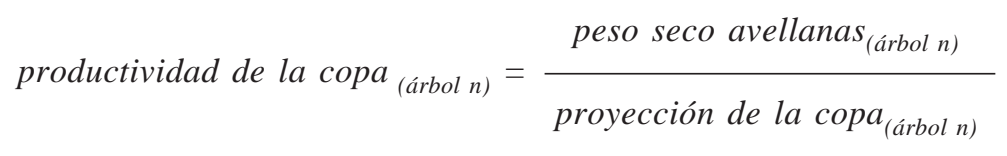

Respecto a este parámetro se destacan los árboles de tipo 1 (productivos grandes) con 282,6 $\mathrm{g} / \mathrm{m}^{2}$ de avellanas (cuadro 2), mientras que los de tipo 3, 4 y 5 alcanzan productividades de copa inferiores de 38,2, 39,4 y 9,2 $\mathrm{g} / \mathrm{m}^{2}$ respectiva- mente. La productividad de los árboles productivos medianos (tipo 2), aunque no difiere significativamente de este último grupo, alcanza un valor superior con $93 \mathrm{~g} / \mathrm{m}^{2}$. Estos árboles, al igual que los de tipo 1 , los favorece su ubicación 


\section{CUADRO 2}

Producción de frutos y productividad de la copa de los distintos tipos de árboles estudiados. Fruit production and crown productivity of the different tree types studied.

\begin{tabular}{|c|c|c|c|c|}
\hline \multirow{2}{*}{$\begin{array}{c}\text { Tipo } \\
\text { de árbol }(*)\end{array}$} & \multicolumn{2}{|c|}{ Producción por árbol } & \multicolumn{2}{|c|}{ Productividad de la copa } \\
\hline & (g/árbol) $(* *)$ & (rango de variación) & $\left(\mathrm{g} / \mathrm{m}^{2}\right)(* *)$ & (rango de variación) \\
\hline 1 & $13.936,4 \mathrm{a}$ & $(1.244-44.347)$ & 282,58 a & $(43,08-505,61)$ \\
\hline 2 & $682,8 \mathrm{~b}$ & $(43-2.168)$ & $93,03 \mathrm{ab}$ & $(7,96-323,58)$ \\
\hline 3 & $2.221,0 \mathrm{~b}$ & $(158-9.950)$ & $38,22 \mathrm{~b}$ & $(4,54-108,19)$ \\
\hline 4 & $314,8 \mathrm{~b}$ & $(113-849)$ & $39,40 \mathrm{~b}$ & $(5,51-125,78)$ \\
\hline 5 & $34,0 \mathrm{c}$ & $(15-74)$ & $9,16 \mathrm{~b}$ & $(1,82-22,77)$ \\
\hline
\end{tabular}

(*) 1: productivos grandes; 2: productivos medianos; 3: grandes de bosque; 4: reserva; 5: renovales.

$(* *)$ expresado en peso seco.

Los valores seguidos de distintas letras presentan diferencia estadísticamente significativa según Prueba de Rangos Múltiples de Tukey, con $\mathrm{P} \leq 0,05$.

aislada, con buena irradiación en los distintos puntos de la copa, factor que podría explicar en parte su mayor producción. Greene (19) señala que la intensidad lumínica y con ello la energía disponible a través de la fotosíntesis, es uno de los principales factores que determinan la diferenciación de yemas florales en especies frutales. Por otro lado, las altas productividades alcanzadas por los árboles de tipo 1 posiblemente deriven de un mejor estado nutritivo de estos avellanos, sometidos a riego complementario y fertilizaciones periódicas. Según Dennis (20), junto con la polinización, el estado nutritivo del árbol frutal juega un rol importante en el cuajado y desarrollo de los frutos.

Tradicionalmente la cosecha de avellanas se efectúa por colectas directas desde el suelo, una vez completada su maduración y caída. En el sustrato los frutos están expuestos a la depredación por roedores $(12,14)$ y a condiciones ambientales cambiantes que pueden afectar su calidad. En este estudio se observaron restos de cáscaras en el suelo, no así dentro de las trampas. También se midieron amplitudes térmicas que superaron $\operatorname{los} 20^{\circ} \mathrm{C}$ y variaciones de humedad relativa entre un 100 y $25 \%$ durante los meses de febrero y marzo 1999. En este contexto, para maximizar la cosecha y optimizar la calidad, es recomendable reducir el tiempo de permanencia de las avellanas en el suelo.

Con la recolección semanal de los frutos de las trampas se obtiene la tasa de caída de avella- nas (figura 2). Se observó que en un mes (16 de febrero a 16 de marzo) cayó más de un $80 \%$ de la producción total de los árboles de tipo 1, 2 y 3. Para los árboles de la reserva (tipo 4), este lapso se redujo a dos semanas ( 23 de febrero a 9 de marzo, figura 2). Tales resultados permiten inferir que en esta latitud es aconsejable realizar la cosecha no más allá de cinco semanas de iniciada la caída de las avellanas.

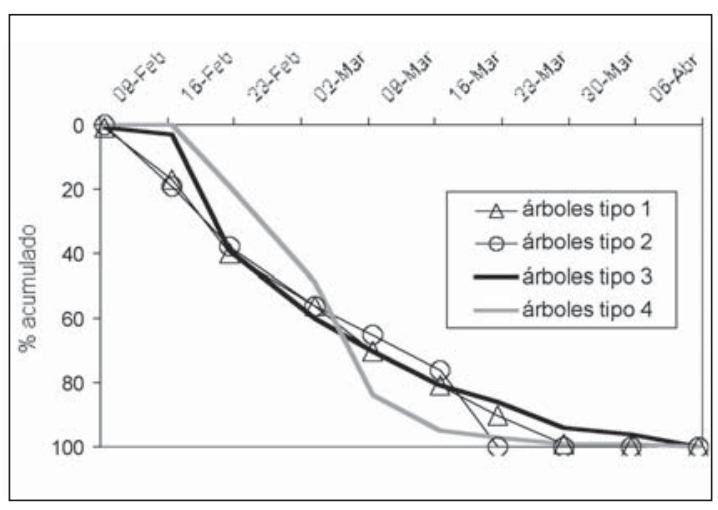

Figura 2. Caída de avellanas maduras en función del tiempo para 4 tipos de árboles estudiados (*), expresada en porcentaje acumulado hasta la fecha.

Mature nut drop in time for 4 tree types studied (**), expressed as cumulative percent up to the indicated date.

(*) 1: productivos grandes; 2: productivos medianos; 3: grandes de bosque; 4: reserva.

(**) 1: large sized productive; 2: medium sized productive; 3: large forest trees; 4 : reserve trees. 
Dimensiones de los frutos. En el cuadro 3 se resumen los principales parámetros de los frutos para cada tipo de árbol. Se encontró una gran variabilidad en el contenido de humedad de las avellanas, contrastando un valor relativamente bajo $(22,82 \%)$ para el caso de los árboles de la reserva (tipo 4) contra valores superiores a $45 \%$ de humedad para los árboles de tipo 1, 3 y 5. Posiblemente la alta densidad de distintas especies arbóreas de gran tamaño en el área de la reserva favorece relaciones de competencia entre los individuos.

El diámetro mayor de los frutos para los distintos tipos de árboles es homogéneo, con valores de 1,89 y $2,03 \mathrm{~cm}$ (cuadro 3 ). Tal resultado se contrasta con la gran variabilidad en su diámetro menor. Las avellanas de los árboles productivos medianos (tipo 2) midieron sólo 1,48 $\mathrm{cm}$ en promedio contra $1,9 \mathrm{~cm}$ de las de los renovales (tipo 5). Estas dimensiones también se tradujeron en volúmenes muy dispares (cuadro 3), alcanzando los frutos de los renovales $3,94 \mathrm{~cm}^{3}$ contra $2,53 \mathrm{~cm}^{3}$ de los productivos medianos. En la figura 3 se observa que los frutos de los árboles de la reserva y renovales (tipos 4 y 5) son más esferoidales con una pequeña diferencia entre el diámetro mayor y el menor, mientras que los frutos de los otros tres tipos de árboles $(1,2$ y 3$)$ son de forma más ovoide, apartándose más marcadamente el diámetro menor del mayor.

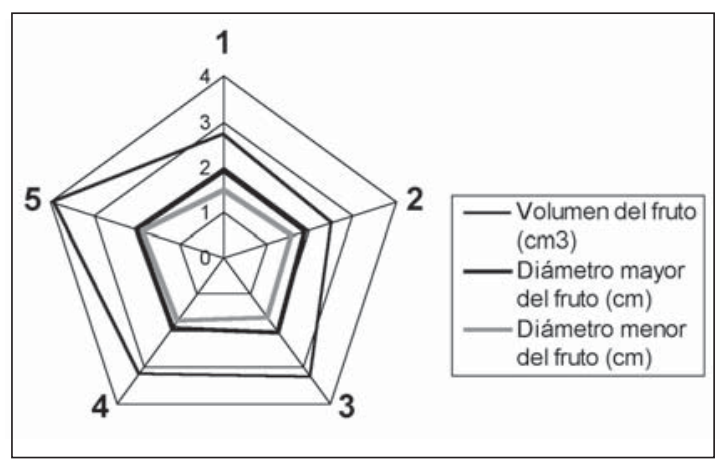

Figura 3. Diámetro mayor, diámetro menor y volumen promedios de frutos de los 5 tipos de árboles estudiados (*).

Mean major and minor nut diameter and mean nut volume of 5 tree types studied $(* *)$

(*) 1: productivos grandes; 2: productivos medianos; 3: grandes de bosque; 4: reserva; 5: renovales.

(**) 1: large sized productive; 2: medium sized productive; 3: large forest trees; 4: reserve trees; 5: young trees.

Estos resultados tienen su explicación parcial en bases fisiológicas. Al aumentar la producción de frutos (número de frutos por unidad de área foliar del árbol), aumenta la competencia entre ellos por los hidratos de carbono disponibles: es mayor la cantidad de destinos (frutos) por unidad de fuentes (hojas) (21). Esto se traduce en una reducción del tamaño individual de los des-

\section{CUADRO 3}

Dimensiones promedio de los frutos de los distintos tipos de árboles estudiados.

Mean nut dimensions of the different tree types studied.

\begin{tabular}{|cccccccc|}
\hline $\begin{array}{c}\text { Tipo de } \\
\text { árbol }(*)\end{array}$ & $\begin{array}{c}\text { Contenido de } \\
\text { agua del fruto } \\
(\%)\end{array}$ & $\begin{array}{c}\text { Diámetro mayor } \\
\text { del fruto } \\
(\mathrm{cm})\end{array}$ & $\begin{array}{c}\text { Diámetro menor } \\
\text { del fruto } \\
(\mathrm{cm})\end{array}$ & $\begin{array}{c}\text { Volumen } \\
\text { del fruto } \\
\left(\mathrm{cm}^{3}\right)\end{array}$ \\
\hline 1 & 45,43 & ab & 1,90 & 1,55 & bc & 2,72 & bc \\
2 & 34,57 & bc & 1,89 & 1,48 & c & 2,53 & c \\
3 & 54,84 & a & 2,03 & 1,64 & bc & 3,28 & ab \\
4 & 22,82 & c & 1,92 & 1,72 & ab & 3,18 & abc \\
5 & 46,00 & ab & 2,02 & 1,90 & a & 3,94 & a \\
\hline
\end{tabular}

(*) 1: productivos grandes; 2: productivos medianos; 3: grandes de bosque; 4: reserva; 5: renovales. Los valores seguidos de distintas letras presentan diferencia estadísticamente significativa según Prueba de Rangos Múltiples de Tukey, con $\mathrm{P} \leq 0,05$. 
tinos (frutos). Por lo tanto, a mayor carga frutal (en nuestro caso los árboles de tipo 1 y 2) tiende a reducirse el tamaño individual de los frutos y a menor carga frutal (árboles de tipo 5) tiende a aumentar el tamaño individual de ellos. Estos argumentos no descartan la posibilidad de que, paralelamente, exista un control genético del tamaño del fruto. En este estudio llamó la atención que algunos individuos se destacaban por producir frutos de mayor tamaño. Además, según reportes de Medel et al. $(6,17,18)$, se logró una selección a favor de este parámetro.

Seguimiento de la transformación de flores en frutos. Según Donoso (22) el ciclo reproductivo de Gevuina avellana dura dos años, iniciándose la diferenciación floral en otoño del primer año. 10 meses después comienza la floración (verano del año 2), culminando un año después (verano del año 3) la maduración del fruto. En esta latitud se observó que la floración se inicia en verano y continúa hasta fines de invierno, evidenciándose los primeros frutos entre junio y julio. Un registro mensual de ramas identificadas permitió seguir la evolución de flores en frutos en tres de las situaciones estudiadas (árboles de tipo 2, 3 y 5).

En la figura 4 puede observarse que en los tres tipos de árboles la floración continuó durante todo el invierno y en el caso de los árboles grandes de bosque (tipo 3) recién se detuvo a mediados de primavera. En las tres situaciones, a inicios del invierno (entre junio y julio), comenzaron a diferenciarse las avellanas, alcanzando a fines del invierno (agosto a septiembre) el mayor número de frutos cuajados. Posteriormente se manifestó una drástica pérdida de frutos formados, manteniéndose a partir de noviembre casi invariable el número hasta su maduración (sólo se manifestó una pequeña caída adicional de avellanas). Como resultado final del total de las flores formadas, sólo un 20,9\% formó frutos (cuadro 4) y de éstas sólo un 22,7\% maduró para el caso de los árboles productivos medianos (tipo 2). Los árboles de tipo 3 y 5 presentaron porcentajes de desarrollo de frutos y de maduración aún inferiores (cuadro 4).

Según Baldini (23), en el caso de árboles frutales sobre un $50 \%$ de las flores pueden afectarse por el fenómeno del "corrimiento", que consiste en la abscisión de flores no fecundadas o que presentan alguna anomalía ovular. Posterior- mente y debido principalmente a la falta de producción de auxinas por parte del embrión, se sucede una fuerte ola de caída de frutitos, conocida en el Hemisferio Norte como "caída de junio" o "junedrop" (23). Luego, puede continuar una ligera caída de frutos, principalmente, debida a factores como estrés hídrico, alteraciones hormonales, carencias nutricionales, agentes patógenos o viento (23). Debido a estos fenómenos se obtiene un porcentaje de cuaje de sólo un $5 \%$ para el caso del manzano y entre 30 y $35 \%$ para el duraznero. El porcentaje de cuaje (cantidad de frutos que maduraron por cada 100 flores formadas) de los avellanos estudiados corresponde a $4,7 \%, 1,6 \%$ y 1,8\% para los árboles productivos medianos (tipo 2), grandes de bosque (tipo 3) y renovales (tipo 5) respectivamente. Debe tenerse en cuenta que recién a partir de junio fueron registradas las flores sobre las ramas, iniciándose en verano la floración. Por lo tanto, es posible que el porcentaje de cuaje sea aún inferior.

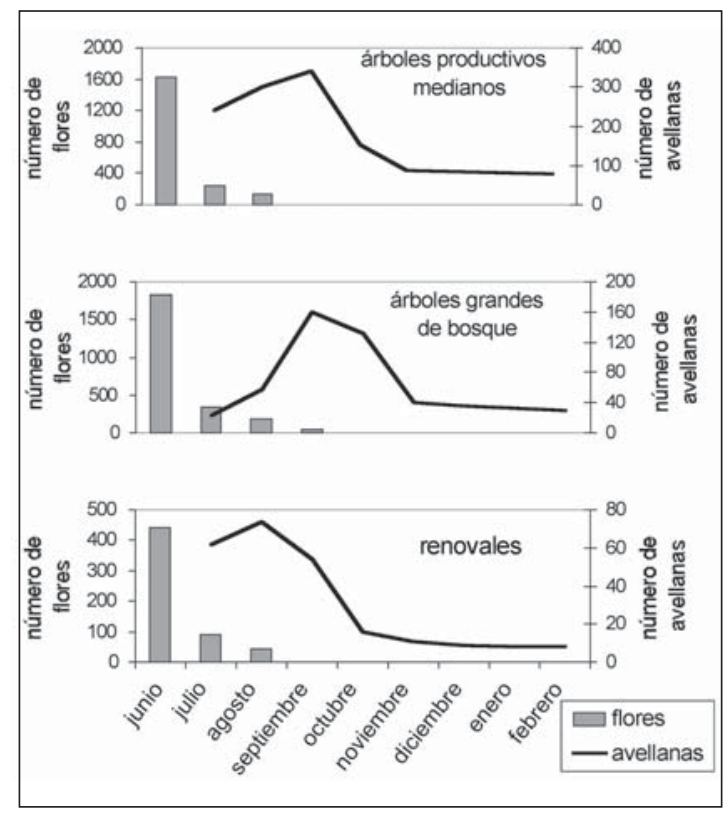

Figura 4. Desarrollo de flores y frutos en árboles productivos medianos, árboles grandes de bosque y renovales.

Flowers and nuts development in medium sized productive trees, large forest trees and young trees. 


\section{CUADRO 4}

Flores y frutos formados sobre cuatro ramas por árbol de tres tipos de los árboles estudiados.

Flowers and fruits formed on four branches per tree of 3 different tree types studied.

\begin{tabular}{|ccccccc|}
\hline $\begin{array}{c}\text { Tipo de } \\
\text { árbol } \\
(*)\end{array}$ & $\begin{array}{c}\mathrm{N}^{\mathrm{o}} \text { inicial } \\
\text { de flores } \\
(* *)\end{array}$ & $\begin{array}{c}\mathrm{N}^{\mathrm{o}} \text { de frutos } \\
\text { formados } \\
(* *)\end{array}$ & $\begin{array}{c}\mathrm{N}^{\mathrm{o}} \text { de frutos } \\
\text { maduros } \\
(* *) \\
(\mathrm{B})\end{array}$ & $\begin{array}{c}\text { \% flores que } \\
\text { formaron } \\
\text { fruto (B/A) }\end{array}$ & $\begin{array}{c}\text { \% frutos que } \\
\text { maduraron } \\
(\mathrm{C} / \mathrm{B})\end{array}$ & $\begin{array}{c}\% \text { de cuaje } \\
(\mathrm{C} / \mathrm{A})\end{array}$ \\
\hline 2 & 1.625 & 339 & 77 & 20,9 & 22,7 & 4,7 \\
3 & 1.835 & 160 & 30 & 8,7 & 18,8 & 1,6 \\
5 & 442 & 74 & 8 & 16,7 & 10,8 & 1,8 \\
\hline
\end{tabular}

(*) 2: productivos medianos; 3: grandes de bosque grandes; 5: renovales.

(**) valores promedio por árbol, resultantes de la suma de las cuatro ramas muestreadas por árbol.

\section{CONCLUSIONES}

De acuerdo a los resultados obtenidos en este estudio, se puede concluir que la producción de frutos de Gevuina avellana en la Cordillera de la Costa de la VII Región varía dependiendo del tamaño y de la posición de los árboles en la vegetación circundante. Las mayores producciones fueron observadas en árboles grandes rodeados de pasturas o cultivos anuales, con cerca de $14 \mathrm{~kg}$ de avellanas por árbol. El estado nutritivo y la iluminación de la copa, son factores que aparentemente incidieron favorablemente en la productividad de estos individuos. Esto sugiere que la especie es factible de incorporarse a manejos silvoagrícolas del secano costero.

Una vez maduros, los frutos caen del árbol dentro de un lapso de 5 a 6 semanas, permitiendo la programación de la cosecha para minimizar pérdidas y mermas de calidad. Aunque el tamaño del fruto se relacionó inversamente a la productividad de los árboles, observaciones en terreno y experiencias anteriores $(6,18)$ indican que se trata de un parámetro con posibilidades de selección.

Se observó que, en esta latitud, la floración se inicia en verano y continúa hasta fines de invierno. Entre la aparición de los primeros frutos hasta su maduración transcurrieron aproximadamente 8 meses. La mayor parte de las flores abortó por razones desconocidas, lográndose porcentajes de cuaje inferiores al $5 \%$.

\section{AGRADECIMIENTOS}

Los autores agradecen la valiosa colaboración de Gabriela Vizcarra y Gladys Ibarra durante el trabajo en terreno y laboratorio.

\section{BIBLIOGRAFIA}

(1) DONOSO, C. Antecedentes sobre producción de avellanas. Bosque (Chile), 1978, vol. 2, No 2, p. 105-108.

(2) RODRIGUEZ, R., O. MATTHEI, M. QUEZADA. Flora arbórea de Chile. Concepción: Ed. Universidad de Concepción, 1983, 408 p.

(3) DONOSO, C. Arboles nativos de Chile. Valdivia: Marisa Cuneo Ediciones, 1994, 116 p.

(4) URBAN, O. Botánica de las plantas endémicas de Chile. Concepción: Soc. Imprenta y Litográfica, 1934, 291 p.

(5) DONOSO, M. El Avellano: un productor múltiple. Chile Forestal (Chile), 1997, vol. 22, $\mathrm{N}^{\circ}$ 251, p. 14-16.

(6) MEDEL, F., R. MEDEL. Gevuina avellana Mol.: características y mejoramiento genético de un frutal de nuez nativo para el mercado internacional. Rev. Frutícola (Chile), 2000, vol. 21, No 2, p. 37-46.

(7) HALlOY, S., A. GRAU, B. Mc KENZIE. Gevuina nut (Gevuina avellana, Proteaceae), a cool climate alternative to macadamia. Economic-Botany (USA), 1996, vol. 50, $\mathrm{N}^{\circ}$ 2, p. 224-235.

(8) DONOSO, C., L. SOTO. Antecedentes sobre producción de avellanas. Bosque (Chile), 1979, vol. 3, No 1, p. 69-70.

(9) DONOSO, C., M. HERNANDEZ, C. NAVARRO. Valores de producción de semillas y hojarascas de diferentes especies del tipo forestal siempreverde de la Cordillera de la Costa de Valdivia obtenidos durante un período de 10 años. Bosque (Chile), 1993, vol. 14, No 2, p. 65-84.

(10) DONOSO, C., B. ESCOBAR, F. UTRERAS, R. REYES, A. ZUÑIGA. Investigación en bosques nativos 
de Chile. Instituto de Silvicultura. Universidad Austral de Chile, 2002, 24 p.

(11) GAJARDO, R. La vegetación natural de Chile. Clasificación y distribución geográfica. Santiago: Editorial Universitaria, 1994, $165 \mathrm{p}$.

(12) DONOSO, C. Ecología forestal. El bosque y su medio ambiente. Santiago: Editorial Universitaria, 1992, $369 \mathrm{p}$.

(13) HARPER, J. L. Population biology of plants. London: Academic Press inc., 1983, $892 \mathrm{p}$

(14) MURUA, R., L. A. GONZALEZ. Producción de semillas de especies arbóreas en la Pluviselva Valdiviana. Bosque (Chile), 1985, vol. 6, No 1, p. 15-23.

(15) MOORE, P. D., S. B. CHAPMAN. Methods in Plant Ecology. England: Blackwell Scientific Publications, 1986, 589 p.

(16) MUÑOZ, M. Análisis comparativo de la producción frutícola en racimos de Gevuina avellana Mol. en tres sitios de la Cordillera de la Costa de la VII Región de Chile. Memoria de título. Universidad de Talca, 2002, 109 p.

(17) MEDEL, F., C. DONOSO y S. HALLOY. Variación en Gevuina avellana Mol. In: IPINZA, R., L. GALLO, A.
PREMOLI, C. DONOSO. Variación Intraespecífica en las especies arbóreas de los bosques templados de Chile $y$ Argentina. Santiago: Editorial Universitaria, 2004, $426 \mathrm{p}$.

(18) MEDEL, F. Genetic and production improvement of Gevuina avellana Mol. in Chile: selected clones for nut production. Nucis-Newsletter, 2001, No 10, p. 17-20.

(19) GREENE, D. Flower development. In: MAIB, K., P. ANDREWS, G. LANG, K. MULLINIX. Tree fruit physiology: growth \& development. Washington: Good Fruit Grower, Yakima. 1996. p. 91-98.

(20) DENNIS, F. Fruit set. In: MAIB, K., P. ANDREWS, G. LANG, K. MULLINIX. Tree fruit physiology: growth \& development. Washington: Good Fruit Grower, Yakima, 1996, 99-106

(21) TAIZ, L., E. ZEIGER. Plant physiology. California: The Benjamin/Cummings Publishing Company, 1991, 565 p.

(22)DONOSO, C. Bosques templados de Chile y Argentina. Santiago: Editorial Universitaria, 1993, 484 p.

(23) BALDINI, E. Arboricultura general. Madrid: Ediciones Mundi-Prensa, 1992, 382 p. 